\title{
Spatial Interpolation of Annual Precipitation in South Africa - Comparison and Evaluation of Methods
}

\author{
M. Coulibaly and S. Becker, University of Wisconsin Oshkosh, Department of Geography \\ and Urban Planning,
}

\begin{abstract}
Data from 545 rainfall gauges were used to interpolate the spatial distribution of annual rainfall in South Africa. Several spatial interpolation methods (inverse distance weighting (IWD), ordinary kriging, universal kriging, cokriging) were tested by variation analyses and cross-validation to determine the most suitable one. The best results were achieved by ordinary kriging, whereby the setting of the parameters was determined through sensitivity analyses. The median of the errors turned out to be $61 \mathrm{~mm}(11 \%)$. The interpolation errors were generally small for the interior of the country and high for coastal and mountainous regions.
\end{abstract}

Keywords: Spatial interpolation, data, evaluation, South Africa

\section{INTRODUCTION}

Precipitation data are important to many applications in hydrology or agriculture. However, no matter how dense the network of measuring stations in a region, there will always be many locations with no available precipitation data. Therefore it is necessary to estimate point rainfall at unrecorded locations from values at surrounding sites (Goovaerts, 1999).

A number of methods have been used in the past for the spatial interpolation of precipitation. Spatial interpolation methods are techniques that predict the value at a given location by using values from sample points. For each computation the values of measured points are weighted depending on their locations. There are many spatial interpolation methods including density estimation, inverse distance weighted method, thin-plate splines method, etc. All methods share the underlying assumption that sample points that are closer to the interpolated location will influence the interpolated value more strongly than sample points which are further away. A key difference among these approaches is the criterion which is used to weigh the values of the sample points. Criteria may include simple distance relations (e.g., inverse distance methods), minimization of variance (e.g., kriging and co-kriging), minimization of curvature, and enforcement of smoothness criteria (splining) (Hartkamp et al., 1999).

The interpolation methods can be classified in two major groups depending on the nature of the function that is used to interpolate the values. A first group of spatial interpolation methods uses mathematical formulas and sample point values to estimate unmeasured values at any point across a given surface. The weight that is assigned to each known value for the interpolation of unknown values depends only on the distance between sample point and location of the interpolated point. These methods are grouped as deterministic and they can be global (making use of all of the sample points) or local (using a limited number of sample points in the vicinity of estimated value's location). When it comes to interpolating precipitation data, the simplest approach consists of assigning the record of the closest gauge to the unsampled location (Thiessen, 1911; Goovaerts, 1999). This method, also referred to as the "nearest neighbor" method (Hartkamp 
Spatial Interpolation of Annual Precipitation in South Africa-Comparison and Evaluation of Methods

et al., 1999), estimates data by drawing a polygon around each gauge of influence with the boundaries at a distance halfway between gauge pairs, hence the name Thiessen polygon for the technique (Goovaerts, 1999). Another method estimates the precipitation data for an unsampled location as a weighted average of the data of surrounding stations. The weights for this method are reciprocal to the squared distances between the location and the surrounding stations (Bedient and Huber, 1992). This method is referred to as the Inverse Distance Weighted method (IDW). The Thiessen polygon method and the IDW technique do not allow the consideration of external factors, such as topography, that can affect the precipitation at a specific location. Not only there is a multitude of factors (moisture content, altitude, pressure, orographic effect, variation of wind speed and direction, proximity of shoreline, etc.) that affect the distribution of rainfall, but also these factors have random and local variation that could hardly be comprehensively taken into account by any existing deterministic method. McCuen (1998) suggests the use of the isohyetal method, which takes into consideration factors that affect the catch at each measuring station to draw lines of equal rainfall depth (isohyets). The amount of precipitation at any location is then estimated by interpolation within the isohyets (Goovaerts, 1999).

A second group of methods is called geostatistical or stochastic methods. These methods are increasingly preferred because they capitalize on the spatial correlation between neighboring observations to predict attributed values at unsampled locations (Goovaerts, 1999). They are not simply based on an estimation of the unknown value as a function of the distance. In addition to that they implement the function of unknown spatial autocorrelation between the values of the sample points. The Kriging method, invented by the South African DG Krige, uses the sample points values and relative positions to model the statistical spatial (auto)correlation with a function that best expresses the variation of the known values. The statistically derived function is then used to compute the value for each location in a region. A detailed discussion of the methods can be found in Hartkamp et al. (1999). Goovaerts (1999) claimed that a major advantage of kriging over simpler methods is that sparsely sampled observations of the primary attribute can be complemented by secondary attributes that are more densely sampled.

Several authors (Tabios and Salas, 1985; Phillips et al., 1992, Dubrule 1983; Burrough and McDonnell 1998; Stein and Corsten 1991; Laslett 1994) have shown that geostatistical prediction techniques (kriging) provide better estimates of rainfall than conventional methods. Hutchinson and Gessler (1994) advocate for the advantages of the "Thin Plate Smoothing Splines". Hartkamp et al. (1999) did not find significant differences after applying various spatial interpolation techniques (inverse distance weighted averaging, thin plate smoothing splines and co-kriging) to precipitation data in Jalisco, Mexico. Burrough and McDonnell (1998) concluded that most interpolation techniques give similar results when data are abundant. This finding is confirmed by Dirks et al. (1998). Nalder and Wein (1998) found that a method named GIDS (gradientplus-inverse distance squared) came up with better results for their study in Canada than all other methods including various kriging methods. This finding was somewhat put into perpective by the study of Price et al. (2000). Borga and Vizzaccaro (1997) found similar results when they compared kriging and multiquadratic surface fitting for various gauge densities. Collins and Bolstad (1996) and Hartkamp et al. (1999) stated that splining provides better results when dense, regularlyspaced data are available and is not recommended for irregular spaced data. Lahmer et al. (1999) compared the outcomes of calculations without station specific correction ('uncorrected Kriging') with an approach that included station specific correction ("corrected Kriging' - differentiation for four precipitation types). They come to the conclusion that the latter method appears to be superior but admit that the results strongly depend on the study region.

The use of elevation data as a source of secondary information has been used by Daly et al. (1994) by estimating the missing data by a rainfall versus elevation regression analysis. Hevesi et al. (1992 a,b) and Goovaerts (1999) incorporated elevation into the estimation of precipitation by cokriging. 


\section{Region and Data}

The aim of the study is to investigate several methods regarding the calculation of the spatial distribution of rainfall over South Africa. Southern Africa's location in the subtropics causes it to be affected by circulation systems of the tropics and the temperate latitudes (Preston-Whyte and Tyson, 1993). Fine weather is usually associated with the influence of the large subtropical anticyclones which are centered over the subcontinent. Disturbances which cause precipitation are either disturbances in the tropical easterly flow (easterly waves and lows) or in the westerly flow (westerly waves, cut-off lows, southern meridional flow, ridging anticyclones, or cold fronts). Another important factor that affects the precipitation pattern over South Africa is the location of the escarpment that separates the low lying coastal areas from the "highveld" and causes orographically induced precipitation on the oceanward and rain-shadow regions on the landward side.

The evaluation is based on monthly precipitation data of 545 weather stations in Southern Africa. Data were supplied by the South African Weather Service and supplemented by the EarthInfo GHCN Global Climate database as well as the FAOCLIM database (FAO, 2002). The distribution of the weather stations can be seen in Figure 1.

Most data are available for the climate normal period of 1961 - 1990. However, data for this period were not available for all stations. Therefore, a second period was considered. Figure 2 shows the relative frequency of available station data in the two climate normal periods 1961 - 1990 and 1931 - 1960. It can be seen that precipitation records are available for approximately $60 \%$ and $70 \%$ of the 545 stations for at least 25 years of the climate normal periods. The cumulative functions show that data of at least 20 years were available for approximately $80 \%$ of the stations etc. To fill the data gaps it was necessary to consider data of both climate normal periods. Figure 3 shows the absolute and relative difference between 1931 - 1960 and $1961-1990$ average annual precipitation data. This calculation is based on 163 stations, for which data are available from 1931 to 1990 . The significantly different summer and winter rainfall seasons, which are a result of the aforementioned geographical location of Southern Africa was the motivation to distinguish between summer and winter precipitation. It becomes obvious that $27 \%$ of the stations show a deviation of less than $10 \mathrm{~mm}$ in winter between the periods. The cumulative function for the absolute deviations reveals that more than half of the stations show deviations of less than $20 \mathrm{~mm}$ in winter. In terms of relative deviations, the deviation is less than $5 \%$ for $40 \%$ or less than $10 \%$ for $70 \%$ of the stations in winter. The deviations for summer were generally slightly higher with regard to the relative deviations and lower with regard to the absolute deviations. The median for winter deviations is $6.6 \%$ or $18 \mathrm{~mm}$, the values for summer deviations are $7.7 \%$ and $10 \mathrm{~mm}$, respectively. The 90th percentile for the average winter deviations amounts to $17.9 \%$ $(52 \mathrm{~mm})$ and $19.0 \%(35 \mathrm{~mm})$ for average summer deviation. These deviations appeared to be acceptable, considering the fact that the average winter precipitation for all locations in South Africa varies between 13 and $1600 \mathrm{~mm}$ (19 and $1318 \mathrm{~mm}$ for summer precipitation). The data gaps of the 1961-1990 climate normal periods were consequently filled with precipitation data from the previous period. The temporal homogeneity of the data was not examined specifically because we only used 30-year averages and possible outliers could be identified by the subsequent spatial analyses.

\section{Data exploration}

The application of stochastic interpolation methods (kriging) requires the specification of a certain procedure (i.e. ordinary, simple, or universal kriging) as well as several parameters. For this purpose it is necessary to elucidate several aspects of the data, in particular those which will influence the selection of parameters. Various exploration methods were used to analyze the spatial variability of the relevant and available input variables. Spatial components of the distribution of annual precipitation and elevation data of the sample points were analyzed, using the exploration tools provided in the geostatistical analysis extension of ArcGIS. The analyses provided information on global and local spatial trends and thereby possible reasons for 
Spatial Interpolation of Annual Precipitation in South Africa - Comparison and Evaluation of Methods

directional components in the data sets.

The application of the kriging method for the spatial interpolation of data assumes a normal distribution. The histogram of the precipitation data (Figure 4) shows that the data are not entirely normally distributed. The distribution is characterized by a skewness of 0.7 and a kurtosis of 4.7. The Q-Q-Plot (Figure 5a), based on the normal distribution, shows that most sample points are close to the line of best fit except for the extremely low or high values. The sample stations that are associated with the deviating values in the upper part of the distribution are located at the northeastern part of the escarpment and are obviously associated with orographically induced rainfall (Figure 5b). The sample stations that are associated with the deviating values in the lower part of the distribution are located in the dry western Karroo region. It can be seen from the Q-Q-plot that - apart from these relatively few stations - most of the other stations are close to the normal distribution. Therefore it appears to be appropriate to use the kriging method in the spatial analyses.

The degree to which precipitation values of adjacent stations varies is referred to as autocorrelation, and could be affected by directional features such as wind direction or the orientation of mountain ranges. Therefore, the precipitation data was examined for directional autocorrelation, both at the overall level and among neighboring stations. The examination of the overall spatial trend analysis (Figure 6) revealed a relatively clear east-west trend as well as less pronounced north-south trend. This aspect of the data suggested the application of a first or second order polynomial trend removal to highlight local variations.

Pairs of points at the same distance apart and in same direction from one another are grouped (binning) and the average of their semivariance (half of the difference squared) are stored in bins. These bins are stored in a graph called the semivariogram surface. In the semivariogram surface the values are colored coded, which allows an overall (global) trend (northeastsouthwest ellipse) to be seen (Figure 7). The directional trend of the semivariogram surface conforms to the one suggested by the spatial trend analysis in figure 6 .

In the semivariogram cloud (Figure 8a) each dot represents the average half of the difference squared (semivariance) for all pair with same direction and distance. The $\mathrm{X}$-axis represents the distance between two locations, while the Y-axis represents the semivariance. A set of points highlighted in the lower left part of the graph were selected to analyze trends in the local spatial autocorrelation figure (Figure 8b), because they are closer in distance and they have a lower semivariance.

\section{Evaluation of Spatial Interpolation Methods (cross validation)}

Given that there is a multitude of factors (elevation, orographic effects, prevailing wind direction, proximity of the oceans, etc.) that will have an impact on the distribution of the rainfall, deterministic models (such as IDW) do not appear to be the most promising approaches for the spatial interpolation of the latter. The algorithms of the kriging methods appear to be clearly better suited for this purpose. Both approaches have been tested with various parameter settings. The quality of the interpolation methods are assessed by evaluating various prediction errors that are derived from the crossvalidation process. That process is essentially based on the fact that the autocorrelation model is derived for the values and locations of all sample points. Then each sample point value is iteratively omitted in the model while estimated value is computed for that same point location. The estimated value is then compared to the measured one for error estimation. Five types of prediction errors presented by Johnston et al. (2001) were taken into consideration.

Provided

$\mathrm{n}$ is the number of samples,

$\mathbf{S}_{\mathbf{i}}$ is a given sample location,

$\hat{\mathrm{E}}\left(\mathrm{S}_{\mathrm{i}}\right)$ is the prediction value for cross-validation, 
$\mathbf{Z}\left(\mathrm{S}_{\mathrm{i}}\right)$ is the observed value, and

$\hat{\mathbf{U}}\left(\mathrm{S}_{\mathrm{i}}\right)$ is the prediction standard error for a given location,

these prediction errors are expressed as follows:

Mean,

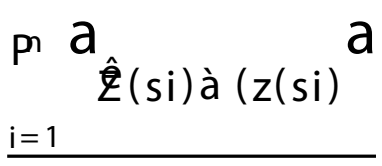

$\mathrm{n}$

Root-Mean-Square (RMS),

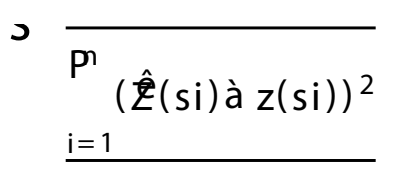

n

Average Kriging Standard (AS),

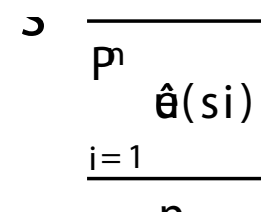

Standardized Mean (SMean),

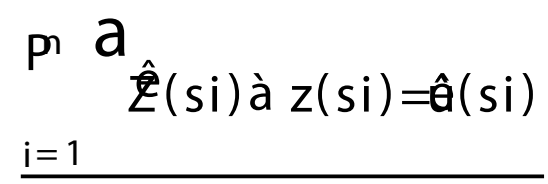

$\mathrm{n}$

Standardized Root-Mean-Square (SRMS)

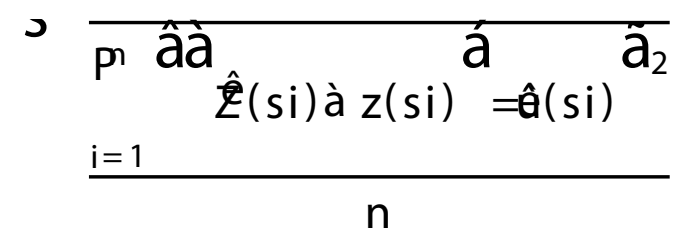

According to Johnston et al. (2001) a good interpolation will yield a SMean error close to the value
"0" (expressing the fact that the prediction error are unbiased), a small RMS error (expressing the similarity between the measured and estimated values). The variability of the predictions from the measurement values is also assessed by comparing the AS error to the RMS one or by comparing the SRMS error to the value one (1): the AS error should be close to the RMS error, and the SRMS error close to "1" for a good estimation of the variability in the prediction. The different prediction errors were assigned weight as follows:

\begin{tabular}{|l|l|}
\hline Criterion (C) & Weigths $(\mathrm{W})$ \\
\hline $\mathrm{C} 1=\mathrm{RMS}$ & W1 $=3$ \\
\hline $\mathrm{C} 2=$ SMean & W2 $=2$ \\
\hline $\mathrm{C} 3=\mathrm{AS}-\mathrm{RMS}$ & $\mathrm{W} 3=1$ \\
\hline $\mathrm{C} 4=1-\mathrm{SRMS}$ & $\mathrm{W} 4=1$ \\
\hline
\end{tabular}

The rationale for the weight assignment is to privilege the error directly related to the proximity of the prediction values to the measured ones (RMS). That error was given a weight of three (3). The other errors were weighted as follows: a weight of two (2) for the SMEAN and a weight of one (1) for each of the errors associated with the estimation of variability in prediction.

Two approaches were applied to evaluate the overall quality of the interpolations. The first approach is based on simply calculating the sum of the ranks (Rank Sum) with no consideration for the weight. For the second approach, the relative importance of the four criteria was incorporated by assigning the weights (W1 - W4) to each of them. The resulting overall criterion for that approach is the sum of the weighted ranks (Rank WSum). In either approach, the four criteria were consequently ranked for all of the interpolation methods and associated parameters.

After applying the IDW and the various kriging methods (Ordinary Kriging, Ordinary Cokriging, Simple Kriging, Universal Kriging) the results of the different approaches were evaluated. A preliminary test run and cross-correlation based on default parameters for the kriging methods were used to compare the applicability of the methods. First and second order trend removal was applied and the anisotropy was considered based on the insights from the exploratory analysis. Table 1 shows the results of the experiment. 
Spatial Interpolation of Annual Precipitation in South Africa - Comparison and Evaluation of Methods

The only parameter that is available to evaluate the IDW approach and compare it to the kriging methods is the mean error. It can be seen that the mean absolute mean error is considerably higher for the IDW approach in comparison to most kriging methods. The best results are provided by Ordinary Cokriging and Universal Kriging. However, the top three kriging methods used with their default parameters have very close scores $(13,14$, and 15 for the RankSum column and 34, 35 and 37 for the RankWSum column).

A sensitivity analysis was conducted to further test the reliability of the Ordinary Cokriging and Universal Kriging approaches. The variable parameters for these examinations were the trend removal approaches, the number of sample involved in computation and the semivariogram model. The comparison process was consistently applied to select methods and parameters and evaluate different combinations of alternatives. In most cases, the simple ranking ("Rank" column) and the weighted ranking ("RankW" column) provided close or even identical results. The results of the sensitivity analyses were used to find the "optimum" interpolation approach.

The first analysis (Table 1) shows that both trend removal and anisotropy considerations led to increased error although the results of the data exploration had suggested the application of these approaches. A systematic iterative first order global trend removal from $100 \%$ to $30 \%$ in $5 \%$ increments was done to analyze the impact on the resulting errors.

The differences between the errors are not significant for most cases. Relatively large errors were observed for higher trend removal $(30 \%-45 \%)$ as well as for the $60 \%$ and $65 \%$ removal. Both ranking approaches (Rank and RankW) provide similar results.

It is noteworthy that the resulting errors after iterative global trend removals are entirely different for the Universal Kriging approach. The smallest errors are generally obtained below $65 \%$ trend removal with an optimum at $30 \%$.

The comparison of the errors associated with the trend removal approaches with the kriging methods without trend removal reveals that even an optimized trend removal approach will not provide better results. It is therefore concluded that trend removal, even though suggested by the exploratory analysis, should not be applied to interpolate precipitation data for the region.

The number of samples that are incorporated in the interpolation of one value is generally an important parameter for the accuracy of the calculation. To find the optimum number of samples the analysis was repeated with between 2 and 70 samples used in the estimation of each interpolated point. The lack of local autocorrelation trends (Figure 8) suggested the choice of a circular shape for the sample selection.

The lowest errors were achieved with a choice of 11 or 13 samples, although no major improvements seem to be achieved above 5 samples. The top ranking numbers of samples were different for the two classifications (13 for Rank and 11 for RankW). Although the SRMS values are more or less in the order of magnitude of the kriging methods with default values, there is a significant reduction of the SMean at and around the computation with 13 samples. The variation in the number of samples has a positive impact on the interpolation and the values 13 and 11 were retained for the final calculations.

Table 2 shows the errors of the interpolation associated with the choice of the semivariogram model. The differences in errors between the various approaches are considerable. The best results according to Rank and RankW classification were provided by the circular model and the exponential model.

From the above set of analysis, it is concluded that Ordinary Cokriging and Universal Kriging, based on 11 or 13 samples chosen by the spherical and circular semivariogram models would results in the smallest error for the interpolation of annual rainfall in the South Africa. The optimum trend removal (30\% for first order) was also considered. These methods/parameters were combined in 12 possible alternatives (Table 3). The interpolation results of the 12 combinations are shown 
in Table 4.

The cross-correlation errors of all twelve combinations are significantly lower than the ones which resultfrom themethodbasedondefaultparametersettings (see Table 1). For universal kriging, for instance, the absolute value of the standardized mean error improved from 0.0059 (with default parameters) to 0.00008 (with selected parameters). Although all four combinations do not show major differences regarding the errors, we come to the conclusion that the combinations 3 and 4 provide the most accurate results. Universal Kriging is the method of choice according to the weighted ranking method whereas ordinary cokriging appears to be better according to the un-weighted ranking method. The spatial interpolation of annual rainfall in South Africa, according to the Universal Kriging method and the parameter combination 4, is shown in figure 9 .

A closer look at the errors associated with the spatial interpolation is provided in the following figure. The validation of the ordinary cokriging interpolation results was done by cross correlation between measured and interpolated station data. The relative frequency of stations with errors below certain thresholds can be taken from figure 10 .

Almost half of the stations show interpolation errors of less than $50 \mathrm{~mm}$. The median of the absolute errors is $61 \mathrm{~mm}$. This appears to be an acceptable value given the median of the measured values is $623 \mathrm{~mm}$ (arithmetic mean: $626 \mathrm{~mm}$ ). The frequency of the stations drops sharply as the maximum error of the interpolation increases and hardly any stations show larger errors than $250 \mathrm{~mm}$ (90\% percentile: $233 \mathrm{~mm}$ ). In terms of relative errors we find that almost half of the stations show less than $10 \%$ error. The median of relative errors is $11 \%$. Stations with an interpolation error larger than 50\% are clearly exceptions (90\% percentile: $41 \%)$.

Absolute interpolation errors on the central highveld are generally relatively small. Apart from a few exceptions the absolute errors are below $100 \mathrm{~mm}$ (Figure 11, right). It is no surprise to find small absolute errors in the northwestern low rainfall region; however, it is noteworthy that the absolute errors are also small for most of the stations in rest of the country. It is clearly visible that the highest absolute interpolation errors occurred in the coastal regions along and seaward of the escarpment as well as in the south-western Cape Region, Lesotho and Swaziland. These are obviously the regions that are characterized by marked orographic features. The Cape mountain chains cause considerable small scale rainfall differences, depending on the elevation and the exposition of the gauge locations. Similar effects are responsible for the poor results in the other regions mentioned above. The spatial distribution of relative errors (Figure 11, right) reveals considerable interpolation errors in the coastal Atlantic region which are mainly due to an underestimation of rainfall amounts. This is an effect that offsets the general spatial trend of the east-to-west rainfall decrease which can be observed for most of the country and is therefore not recognized by the interpolation algorithms. Furthermore, it can be seen that the effect of the rainshadow deserts north of the mountain chains along the Garden Route in the South leads to considerable interpolation errors.

Coastal rainfall characteristics in the west, orographically induced rainfall and the rainshadow effect can quite clearly not be reproduced by the applied interpolation method. The particular location of South Africa with the variety of factors that determine rainfall in the various seasons (see Preston-Whyte and Tyson, 1993) is a major obstacle in detecting coherent spatial trends that would enable the consideration of the orographic effects in the interpolation method.

\section{CONCLUSION}

Spatial interpolation of annual rainfall in South Africa poses various problems due to the complex impact of rain producing systems and orographic features of the country. Testing of various methods and parameters, by comparing and ranking their cross-correlation errors, revealed that either ordinary cokriging or universal kriging method using the circular semivariogram model with thirteen samples provides the overall best results. The overall kriging methods were proven better than IDW. These kriging methods were compared and selected following a procedure 
Spatial Interpolation of Annual Precipitation in South Africa - Comparison and Evaluation of Methods

based on the analysis of cross-correlation prediction errors. Surprisingly, the global trend removal had an overall negative impact on the interpolation methods, even with the optimum value.

Given the quality of the results (significantly low prediction errors), the methodology used was proven efficient and could serve for other sites. Among the semivariogram models, the circular one was the most efficient regardless of the method used. The study showed also how determinant both the number of samples and their selection mode are to the quality of the results. The analysis of cross-correlation errors showed also that results are acceptable for the interior of the country where the overall spatial trend is not disturbed considerably by local effects. Local effects in the coastal and mountainous regions, however, lead to significant interpolation errors. A separate analysis of these regions would be necessary to achieve acceptable results.

\section{References}

Bedient, P.B. and W.C. Huber. (1992) Hydrology and Floodplain Analysis (New York: AddisonWesley).

Borga, M. and A. Vizzaccaro. (1997) On the interpolation of hydrologic variables: formal equivalence of multiquadratic surface fitting and kriging. Journal of Hydrology, 195 (1-4), pp. 160-171.

Burrough, P.A., and R.A. McDonnell. (1998) Principles of Geographical Information Systems (New York: Oxford University Press).

Collins, F.C., and P.V. Bolstad (1996) A comparison of spatial interpolation techniques in temperatire estimation. Proceedings of the Third International Conference on Integrating GIS and Environmental Modeling, Santa Barbara, CA: National Center for Geographic Information Analysis (NCGIA). CD-ROM.

Daly, C., R.P. Neilson and D.L. Phillips. (1994) A statistical topographic model for mapping climatological precipitation over mountainous terrain. Journal of Applied Meteorology, 33 (2), pp. $140-158$.

Dirks, K.N., J.E. Hay, C.D. Stow and D. Harris. (1998) High-resolution studies of rainfall on Norfolk Island Part II: interpolation of rainfall data. Journal of. Hydrology, 208 (3-4), pp. 187-193.

Dubrule, O. (1983) Two methods with different objectives: Splines and Kriging. Mathematical Geology, 15, pp. 245-257.

FAO. (2002) Food and Agriculture Organization of the United Nations - FAOCLIM Ver.2 database - Environment and Natural Resources Service SDRN, Publication No. 9 in the SDRN working paper series, Agrometeorology Group, Rome, Italy.

Goovaerts, P. (1999) Performance Comparison of Geostatistical Algorithms for Incorporating Elevation into the Mapping of Precipitation. The IV International Conference on GeoComputation was hosted by Mary Washington College in Fredericksburg, VA, USA, on 25-28 July 1999.

Hartkamp, A.D., K. De Beurs, A. Stein, and J.W. White. (1999) Interpolation Techniques for Climate Variables. NRG-GIS Series 99-01 (Mexico, D.F.: CIMMYT).

Hevesi, J.A., J.D. Istok and A.L. Flint. (1992a) Precipitation estimation in mountainous terrain using multivariate geostatistics. Part I: Structural analysis. Journal of Applied Meteorology, 31, pp. 661-676.

Hevesi, J.A., A.L. Flint and J.D. Istok. (1992b) Precipitation estimation in mountainous terrain using multivariate geostatistics. Part II: Isohyetal maps. Journal of Applied Meteorology, 31, pp. 677-688.

Hutchinson, M.F., and P.E. Gessler. (1994) Splinesmore than just a smooth interpolator. Geoderma, 
62, pp. 45-67.

Johnston, K., J. M. V. Hoef, K. Krivoruchko, N, Lucas (2001) Using ArcGIS Geostatistical Analyst (Redlands, CA: ESRI).

Laslett, G.M. (1994) Kriging and splines: An empirical comparison of their predictive performance in some applications. Journal of the American Statistical Association, 89, pp. 391-400.

Lahmer, W., Klöcking, B., and Pfützner, B. (1999) Meteorological Input Variables in Mesoand Macroscale Hydrological Modelling. Contribution to the International Conference on Quality, Management and Availability of Data for Hydrology and Water Resources Management Koblenz, 22-26 March, 1999.

McCuen, R.H. (1998) Hydrologic Analysis and Design (N.J.: Prentice Hall).

Nalder I.A. and Wein R.W. (1998) Spatial interpolation of climatic Normals: test of a new method in the Canadian boreal forest. Agricultural and Forest Meteorology, 92 (4), pp. 211-225.

Phillips, D.L., J. Dolph and D. Marks. (1992) A comparison of geostatistical procedures for spatial analysis of precipitations in mountainous terrain. Agricultural and Forest Meteorology, 58, pp. 119-141.

Preston-Whyte, R.A. and Tyson, P.D. (1993) The Atmosphere and Weather over Southern Africa (Oxford: Oxford University Press).

Price, D.T., McKenney D.W., Nalder I.A., Hutchinson M.F., Kesteven J.L. (2000) A comparison of two statistical methods for spatial interpolation of Canadian monthly mean climate data. Agricultural and Forest Meteorology, 101 (2), pp. 81-94.

Stein, A., and L.C.A. Corsten. (1991) Universal kriging and cokriging as regression procedures.
Biometrics, 47, pp. 575 -587.

Tabios, G.Q. and J.D. Salas. (1985) A comparative analysis of techniques for spatial interpolation of precipitation. Water Resources Bulletin, 21 (3), pp. 365-380.

Thiessen, A.H. (1911) Precipitation averages for large areas. Monthly Weather Review, 39 (7), pp. 1082-1084. 